\title{
A Method for Evaluating Ballistic Missile Penetration Operational Effectiveness
}

\author{
Xiaolong Zheng and Lefei Pan \\ Rocket Force University of Engineering, Xi' an Shaanxi 710025, China
}

\begin{abstract}
According to the characteristics of system operational effectiveness and use plan, combat environment and other factors are closely related, which is a typical ballistic missile penetration combat combat system based on evaluation method to establish the effectiveness of combat simulation system based on. In the process of research, the key issues such as general simulation framework, multi-source data fusion and data acquisition are deeply studied, and the solutions are given. The operational effectiveness evaluation of ballistic missile penetration is realized under the premise of considering the factors such as planning and operational environment. The research results can be effectively applied to the evaluation of typical combat system effectiveness.
\end{abstract}

Keywords-ballistic missile; missile penetration; effectiveness evaluation; simulation framework

\section{INTRODUCTION}

Combat effectiveness refers to the given force structure, operational principles, tactics, survivability, vulnerability and threats in the system operational planned or expected environment representative the use of personnel, the overall level of the system to complete the task [1].

From the above definition can be seen in the level of operational effectiveness of weapon system is not fixed, and it is closely related to the use of plans and the expected operational environment and other factors, will present different levels of ability in combat weapon system under the condition of different environment and efficiency. Under the condition of information warfare, the battlefield situation is changing rapidly. According to the actual situation of the battlefield, it is necessary to adjust and change the operation plan promptly and timely, which is one of the necessary ways to improve the combat effectiveness of the weapon system. With the increase of uncertainty of the battle plan and the expected environment, the rapid demand for the evaluation of the combat effectiveness of the weapon system is becoming more and more urgent. The traditional performance evaluation methods and means in dealing with the operational effectiveness evaluation problems, often there is a long period, evaluate the single goal, spending and other aspects of the weapon system is difficult to meet the conditions of information warfare rapid targeted effectiveness evaluation [2]. Therefore, the establishment of effective to adapt to the rapid method of effectiveness evaluation of battlefield environment and combat weapons programs and features, is a necessary means to meet the needs of future information warfare, effectively improve the army's ability to adapt to the battlefield.
Ballistic missile, as the killer weapon of modern war, is an important link in the weapon equipment system. Penetration effectiveness is one of the key components of the operational effectiveness of ballistic missile weapon system, and its evaluation is very complicated. [3]. Due to the close connection between missile penetration capability and operation plan, battlefield environment and other factors, the targeted rapid assessment research has certain representativeness in solving related problems. This paper puts forward to the specific performance of the penetration operation condition assessment ideas, provides a way for fast, according to the evaluation of implementation of penetration effectiveness, but also provide a solution for the similar problems.

\section{EFFICIENCY EVALUATION METHOD ANALYSIS}

On the whole, all kinds of methods and models involving the operational effectiveness of weapon systems at home and abroad mainly include expert evaluation method, test statistics method, combat simulation evaluation method, index method and analytic method, and so on. [4][5]. All of the above operational effectiveness evaluation methods have their own advantages and disadvantages, and each has certain applicable conditions, and they all have the main application objects. However, the rapid and effective performance evaluation is still a hot research topic for the combat problems with timeliness and pertinence. The penetration of ballistic missile combat effectiveness evaluation technology is one of the timeliness and effectiveness of system level higher requirements for assessment, to improve the credibility of the evaluation result, reduce the risk assessment, need to learn from and absorb the advantages of various methods, construct scientific and effective evaluation method.

The operational effectiveness evaluation of missile penetration can be carried out by flight test, but the high test cost and long test period are the main reasons affecting the effectiveness evaluation. At the same time, must also consider the limitations of the test conditions, can not take into account all the operational conditions in the flight test, the evaluation results have general characteristics, it is difficult to meet the operational requirements of [6] under the condition of informationization.

In this paper, the simulation test is taken as the technical means, and the efficiency evaluation model based on Simulation and multiple evaluation methods is proposed. Through the construction of "general missile flight simulation framework, provide a simulation platform for ballistic missile penetration combat assessment; missile defense capability, to 
break through the anti missile defense system missile penetration ability, other factors such as the classification index, based on the consideration of a variety of evaluation methods, evaluation index system of multi dimension performance; Modular Simulation Architecture in order to make the simulation framework, to achieve full digital simulation and semi physical simulation, based on the heterogeneous hybrid simulation experiment design of flight test data and flight test data simulation, which can provide reliable technical support and reliable assessment for simulation and identification; flight test data, evaluation data, expert scoring evaluation data of multi-source and heterogeneous data unified standard the technical evaluation of simulation application, realize the operations of ballistic missile penetration effectiveness of accurate and reliable identification and evaluation; The research results of the project are sorted and integrated to form a simulation test evaluation tool for various types of missiles, so as to provide technical support for the ballistic missile penetration operational effectiveness evaluation.

\section{CONSTRUCTION OF GENERAL SiMULATION FRAMEWORK FOR MISSILE ATTACK AND DEFENSE}

The core of the evaluation is to build a scientific and reasonable simulation system corresponding to the evaluation objectives. It is difficult to adapt all kinds of operational conditions by using a set of fixed systems and models to meet the requirements of different weapon systems, combat environments and operational requirements. In this paper, the idea of building a universal penetration simulation framework is proposed to improve the flexibility and adaptability of the simulation system in the effectiveness evaluation.

To achieve the generality of simulation system, we must first establish a general framework, and then realize the role conversion between scheme and scenario, general and private through modular design. The research process can be divided into three levels: connect the missile penetration simulation module, data flow, using the general framework; on the basis of the establishment of general module and interface specification; general module design code to meet the general requirements, meet according to the specific scenario to replace the general model of complete combat effectiveness evaluation using the corresponding model requirements.

The specific framework of the simulation is shown in the following picture:

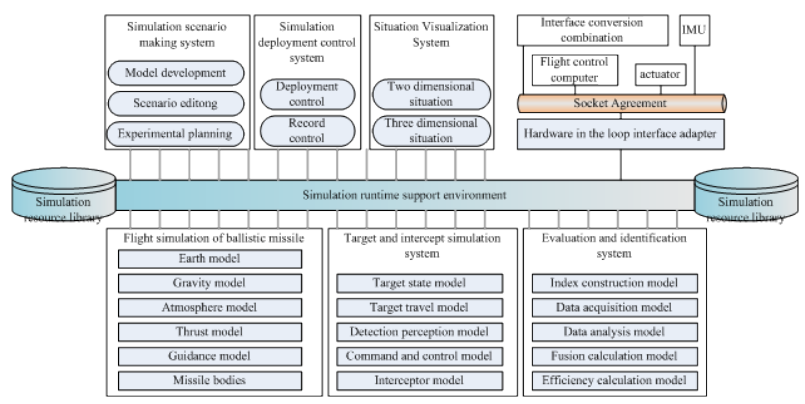

FIGURE I. GENERAL SIMULATION FRAMEWORK OF BALLISTIC MISSILE PENETRATION OPERATION

\section{EFFiCiency Evaluation BAsed on Simulation Test}

The basic idea of evaluation simulation based on Efficiency: constructing the evaluation system of missile system penetration effectiveness based on multidimensional index system, for a given scenario, The simulation system for effective simulation of missile attack process, acquisition and calculation by simulation in the process of assessment of the related data, the integrated use of a variety of performance assessment methods, the corresponding the ballistic missile penetration effectiveness index value, and then judge the missile weapon system penetration efficiency.

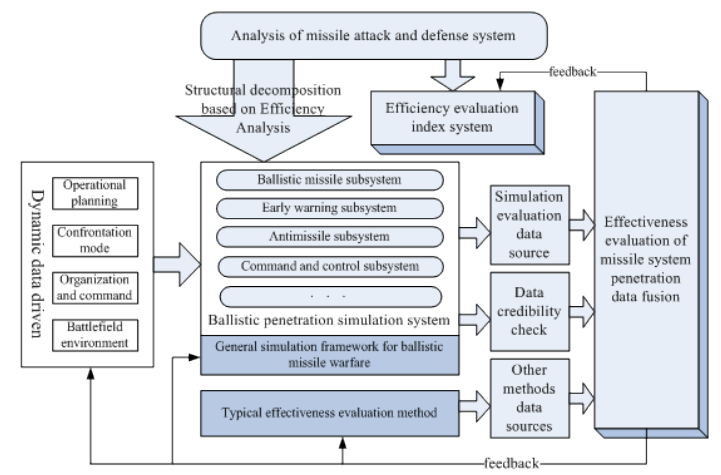

FIGURE II. GENERAL SCHEME FOR EVALUATING PENETRATION EFFECTIVENESS OF BALLISTIC MISSILE

In this paper, the key technologies of missile penetration effectiveness evaluation, such as index construction, evaluation data acquisition and multi-source data fusion, are analyzed and studied.

\section{A. Construction of Efficiency Evaluation Index}

The index system can be regarded as the supporting framework and details of effectiveness evaluation. It is necessary to point out that different operational plans and tasks reflect different operational effectiveness. Therefore, the index system should be a multi-dimensional index system with adaptability, and can give the targeted evaluation index flexibly according to the evaluation requirements.

The index system of missile penetration efficiency evaluation is based on the two indexes which are found and attacked, and the missile weapon itself is also considered. From a broader point of view, the evaluation index system of penetration efficiency should be based on different evaluation purposes to construct multi-dimensional index system reflecting different evaluation needs. The construction of multidimensional index system can reflect the specific operational requirements from different angles. For example, the ratio of penetration can be evaluated in different dimensions to construct the index system of missile penetration probability, missile interception probability and the two ballistic missile penetration effectiveness, different index that reflect the different needs of the mission, and is closely related to [7]. It can be said that the reasonable multi-dimensional effectiveness evaluation index is the basis of evaluation work, and also the premise to get the correct evaluation conclusion.

The process of constructing multidimensional index in assessing the effectiveness of ballistic missile penetration in the 
form of specific indicators, requires a combination of operational plans, operations and the actual situation to determine the integrated simulation system, which focuses on the establishment of rules and guide the selection of indicators are standard, value calculation method on the basis of scientific and reasonable design and evaluation of multidimensional index system compatible with the target the corresponding index, so as to establish a multidimensional index system of reasonable structure, improve the level of.

Specific construction process as shown in the following picture:

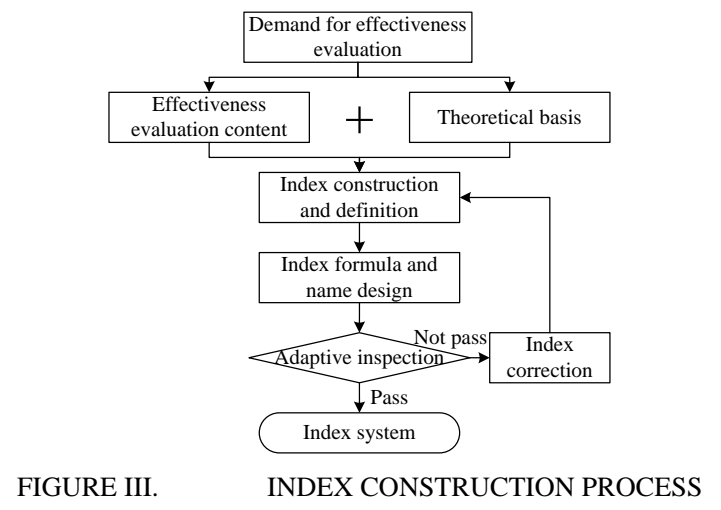

\section{B. Evaluation Data Collection}

Collecting effective simulation data for evaluation is a core work of simulation test method for effectiveness evaluation. The simulation data collection first need to assess needs for refinement of index data needs, and then combined with the simulation model of the index demand reflected, then according to the specific test programs, to collect the required data in the simulation process, and these data used in performance evaluation work. It can be seen that the acquisition of simulation data needs to be coordinated between the evaluation system and the simulation system. The requirements of the evaluation system can be effectively reflected in the form of specific indicators in the simulation system, and the actual state of the simulation system is also the content that needs to be considered when the evaluation system needs to be determined. According to this situation, the simulation test evaluation system can be divided into three subsystems, such as evaluation system, simulation system and data acquisition system, and the old relationship is shown in the following picture:

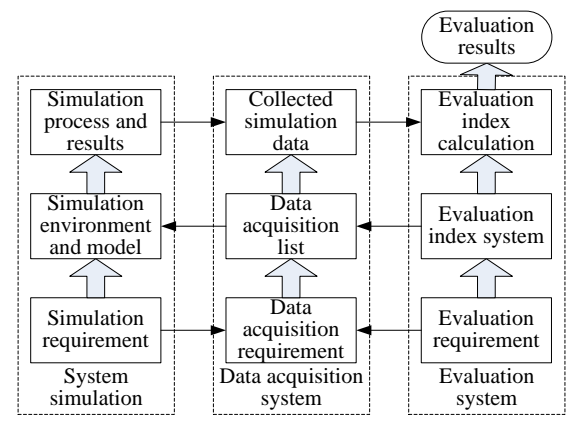

FIGURE IV STRUCTURE DIAGRAM OF SIMULATION DATA ACQUISITION
It can be seen from the relational structure diagram that the data acquisition system is an intermediary for the effective combination of evaluation and simulation. It is mainly reflected in the evaluation requirements of the evaluation system, which needs to be converted into data collection list through the index way, and also puts forward the requirements for the simulation system requirements. The simulation system is to analyze whether the proposed data acquisition list contains the content to be reflected in the simulation system, and can be collected; if the required data simulation system is not directly available, one hand to adjust the simulation model, on the other hand, coordination and evaluation system, re adjust the data collection list. It can be said that the construction of the simulation system is actually to determine the specific simulation purposes according to the needs of the evaluation. According to this idea, through the connection and coordination of the data acquisition system, the data collection list which can be recognized by the evaluation system and the simulation system is obtained. On this basis, according to the confirmed data acquisition list, the data needed for evaluation are collected during the operation of the simulation system, and the effectiveness evaluation is carried out by using the collected data.

\section{Multi source Evaluation Data Fusion Evaluation Method}

The evaluation data acquired by simulation experiment, can't always full of each detail index to reflect the system, so it needs to consider various types of data can be obtained, then the multi-source data fusion assessment method to realize the integration of different data evaluation.

To reduce the risk of assessment and improve the reliability of the evaluation results, it is also necessary to use a variety of evaluation data sources containing different reasonable components. Besides the data collected by the simulation system, the expert's experience information is not only a valuable data source, but also an indispensable complement to the simulation data acquisition when [8] is used to evaluate the penetration efficiency of ballistic missile. Therefore, in the evaluation process, method through information unification and weighted fusion, simulation data based on quantitative and qualitative expression to expression of multi-source data based expert data and other comprehensive assessment, and then get the evaluation conclusion reliable. The data fusion evaluation framework is shown in the diagram below:

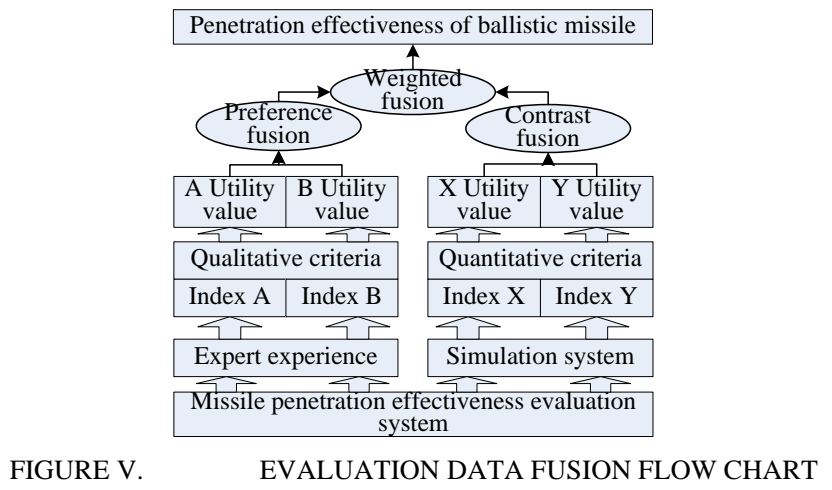




\section{EXAMPLES}

Get to the single stage short-range missile trajectory simulation framework design and general design of XXX-2, anti XXX-3 anti missile defense system against penetration simulation as an example, discusses the application method of the penetration effectiveness of efficiency evaluation and analyzed the result.

Combat simulation scenario is a single attack bomb, missile launch point (XX "XX 'XX" N, XX "XX' XX" E), the target point (XX "XX 'XX" N, XX "XX' XX" E), PAC-3 (XX XX deployment location for ' $\mathrm{XX}$ "N $\mathrm{XX}, \mathrm{XX}$ ' $\mathrm{XX}$ " E), intercept program for the two bar; penetration effectiveness index for penetration probability, the decomposition index at the same time, combined with the model of simulation system, gives the corresponding end index contains 162 content data list, used to collect evaluation data in the simulation process. Part of the acquisition list and data collection is as follows:

\section{TABLE I. COLLECTION AND CALCULATION INDEX LIST}

\begin{tabular}{|l|l|}
\hline \multicolumn{1}{|c|}{ Final index } & \multicolumn{1}{|c|}{ Evaluation data } \\
\hline \multicolumn{1}{|c|}{... } \\
\hline $\begin{array}{l}\text { Discovery time of early } \\
\text { warning satellite }\end{array}$ & $9.02 \mathrm{~s}$ \\
\hline Remote warning time & $191.06 \mathrm{~s}$ \\
\hline Continuous tracking time & $51.04 \mathrm{~s}$ \\
\hline $\begin{array}{l}\text { Ratio of continuous tracking } \\
\text { time }\end{array}$ & $26.7 \%$ \\
\hline Earliest detection distance & $466.34 \mathrm{~km}$ \\
\hline Height of target interception & $511.32 \mathrm{~m}$ \\
\hline Target heel height & $37748.69 \mathrm{~m}$ \\
\hline$\ldots$ & $\ldots$ \\
\hline Guided radar detection time & $419.21 \mathrm{~s}$ \\
\hline Launch time of interceptor & $463.34 \mathrm{~s}$ \\
\hline Interception altitude & $18.34 \mathrm{~km}$ \\
\hline Intercept slant distance & $26.24 \mathrm{~km}$ \\
\hline$\ldots$ & $\ldots$ \\
\hline
\end{tabular}

In addition to the above evaluation data obtained by simulation, the reliability of missile launching and the reliability of missile flight are given by expert experience. Through the standardization and fusion calculation of the evaluation data, the penetration probability of PAC2 and PAC3 two intercept schemes is $84.12 \%$ and $53.63 \%$ respectively.

In accordance with the above method, respectively to position and change the simulation in ballistic shape deployment interceptions and defense system in (launch location) under the premise, to calculate the penetration effectiveness of missile, based on the evaluation results the following conclusions can be drawn: the increase of interceptions can improve the probability of intercept the missile penetration probability; anti missile defense system deployed farther away from the target point, the lower the probability of intercept ballistic penetration probability is higher; higher, the lower the probability of intercept penetration probability is high.

\section{SUMMARY}

The comprehensive evaluation method of missile penetration effectiveness based on Simulation and multiple assessments has the advantages of high flexibility, strong adaptability and economic saving. The establishment of this system and can provide a reliable method available for penetration effectiveness of missile system penetration combat assessment method, which can provide reliable system penetration scheme available for missile combat; at the same time, the demand for new demonstration, the penetration ability of missile weapon system can make innovation theory research, warfare project assessment, key technologies provide verification effective support.

It should be pointed out that in the process of simulation evaluation of penetration efficiency, the current work only considers the terminal defense system antimissile defense system. At present, foreign advanced missile interception technology has been developed for the full range of mesh, anti missile defense system, therefore the performance index and evaluation algorithm needs further and advanced penetration and anti missile technology corresponding to improve technology. This is also the focus of the next step needs to be studied.

\section{REFERENCES}

[1] HUANG Jianxin. System of sytems Effectiveness Analysis and Simulation based on ABMS[D]. Changsha: National University of Defense Technology, 2011, 7-16

[2] SUN Ming, HUANG Jun-qing, MA Ya-long. Research on Methodology of Operational Efficiency Evaluation Based on Combat Simulation Data [J]. Journal of System Simulation, 2013, 25(8):366-371.

[3] HU Yang-guang XIAO Ming-qing KONG Qing-chun GAO Chun-qing et. The Method and Model of Effectiveness Evaluationon Miniature AirLaunched Decoy [J]. Computer simulation, 2016, 33(8):44-48

[4] GAO Chunqing,XIAO Mingqong,Kong Qingchun etc.Research on Effectiveness Optimization Design of Cruise Missile Cooperative Penetration[J]. Computer simulation, 2017, 34(1):48-51.

[5] Chen Zhiqian, Song Junxiao, Yang Chunxin. Review of Combat Effectiveness for Armed Helicopter[J]. Ordnance Industry Automation, 2016, 35(1):12-24

[6] Ma Qingyue. RESEARCH ON COMPREHENSIVE EVALUATION TECHNIQUES FOR WEAPON SYSTEM OF SYSTEMS' OPERATIONAL EFFECTIVENESS [D]. Harbin: Harbin Institute of Technology, 2015, 1-5

[7] HE Shao-tong, XUE Lun-sheng, XU Chen-yang etc. Study on the Evaluation of Operational Effectiveness of Ballistic Missile Penetration [J]. Computer simulation, 2016, 33(9):39-44.

[8] Shen Yanhang, Zhou Zhou. Evaluating Operational Effectiveness of AUAVs inSearch, Classification and Attack [D]. Xi'an: Northwestern Polytechnical University, 2015, 2-10 